\title{
Influence of Polarization on Carbohydrate \\ Hydration: A Comparative Study using Additive and Polarizable Force Fields
}

\author{
Poonam Pandey and Sairam S. Mallajosyula* \\ Department of Chemistry, Indian Institute of Technology Gandhinagar, Simkheda, \\ Gandhinagar, Gujarat, India \\ E-mail: msairam@iitgn.ac.in
}

Phone: +91 - 79 - 32454998. Fax: +91 - 79 - 23972324

Supporting Information 
(a) Gal - TIP3P additive system
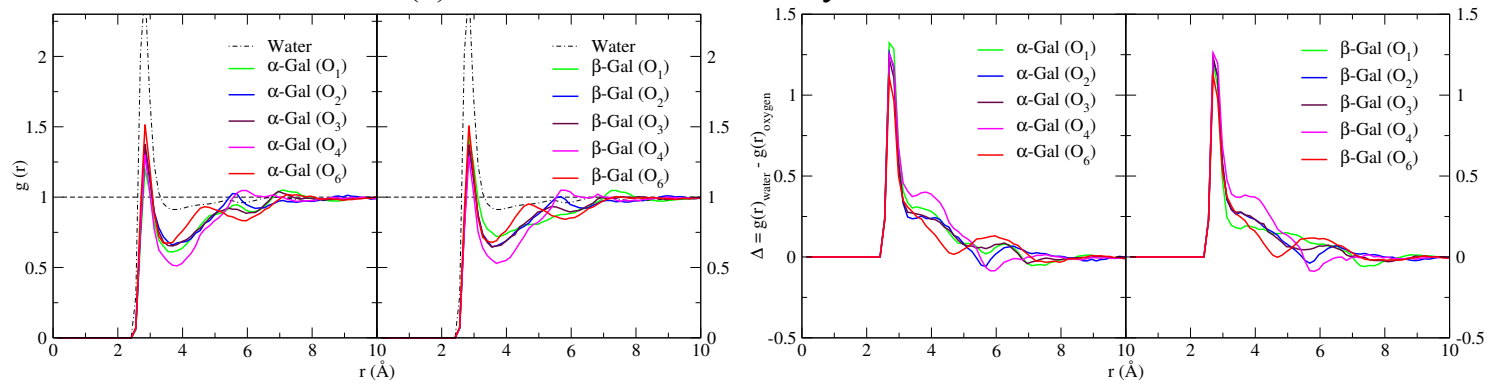

(b) Gal - TIP4P additive system
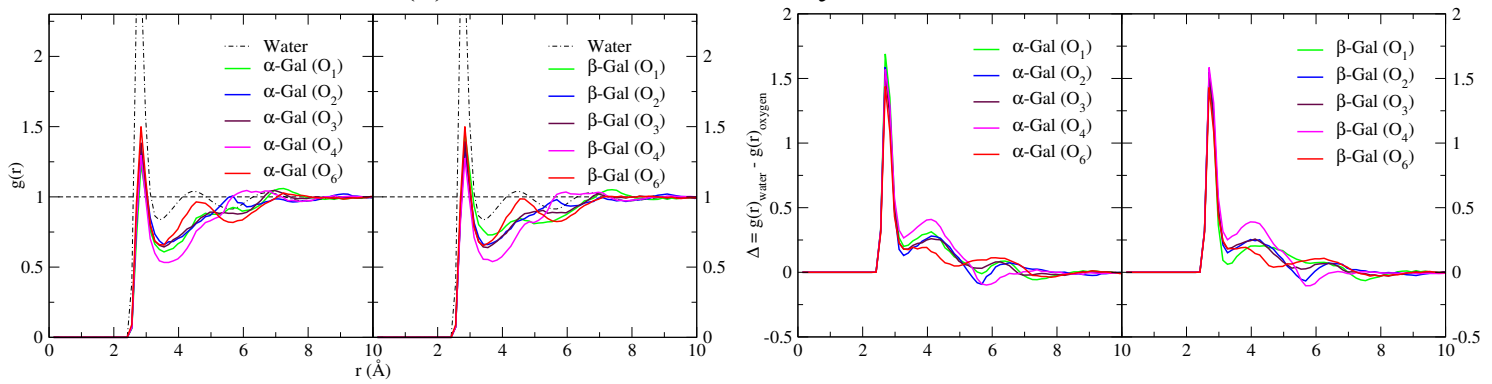

(c) Gal - TIP5P additive system
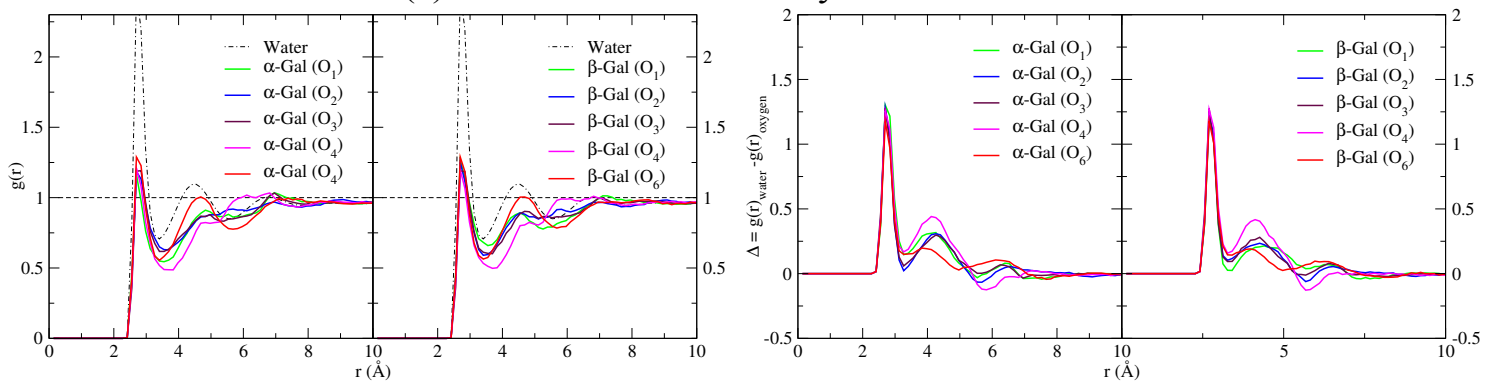

(d) Gal - SWM4 drude system
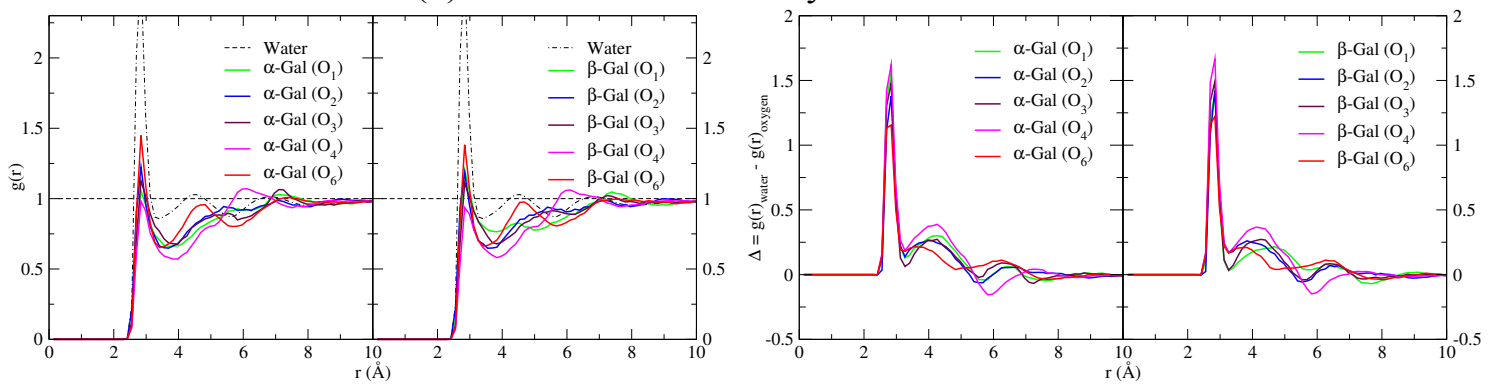

Figure S1: Left Panel: Selected oxygen (carbohydrate) - oxygen (water) radial distribution functions, $g(r)$, from $300 \mathrm{~K}$ simulations of carbohydrate (Gal) in four water models (a) TIP3P, (b) TIP4P, (c) TIP5P and (d) SWM4. Right Panel: Differences $(\Delta)$ between the radial distribution functions. $\Delta$ is calculated as the differences between $g(r)$ of pure water and $g(r)$ of selected oxygen carbohydrate (Gal) - oxygen (water) radial distribution from $300 \mathrm{~K}$ simulations. 
(a) Man - TIP3P additive system
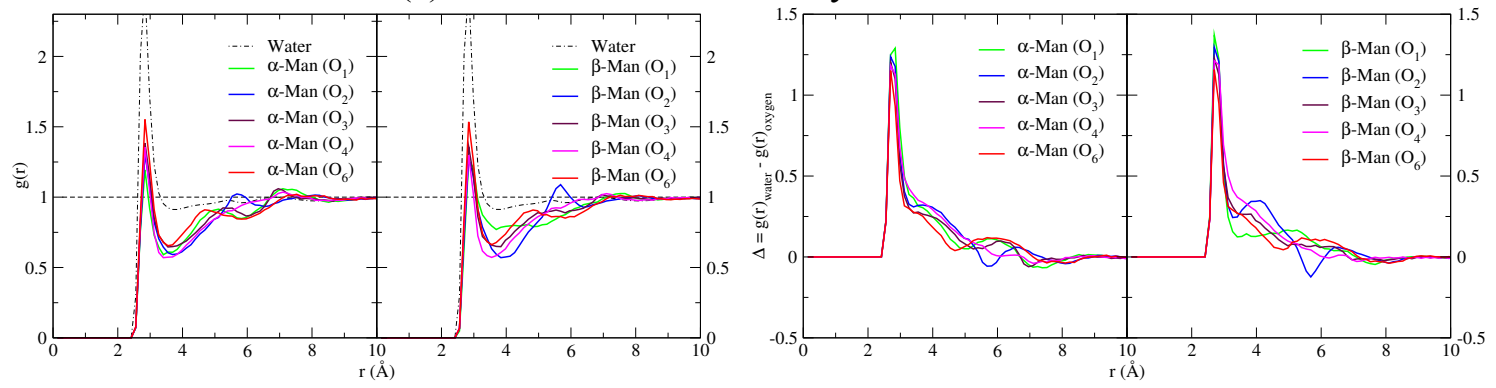

(b) Man - TIP4P additive system
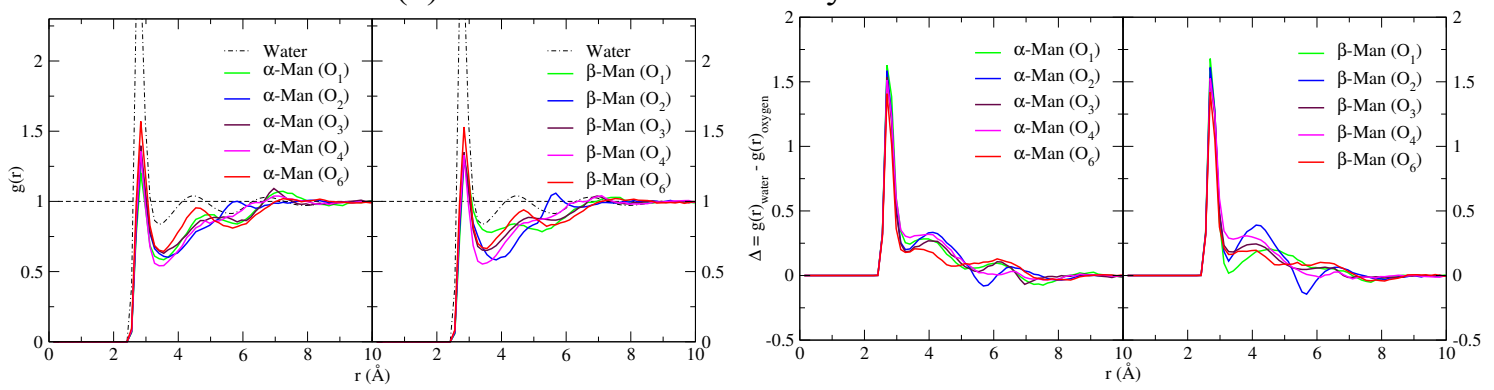

(c) Man - TIP5P additive system
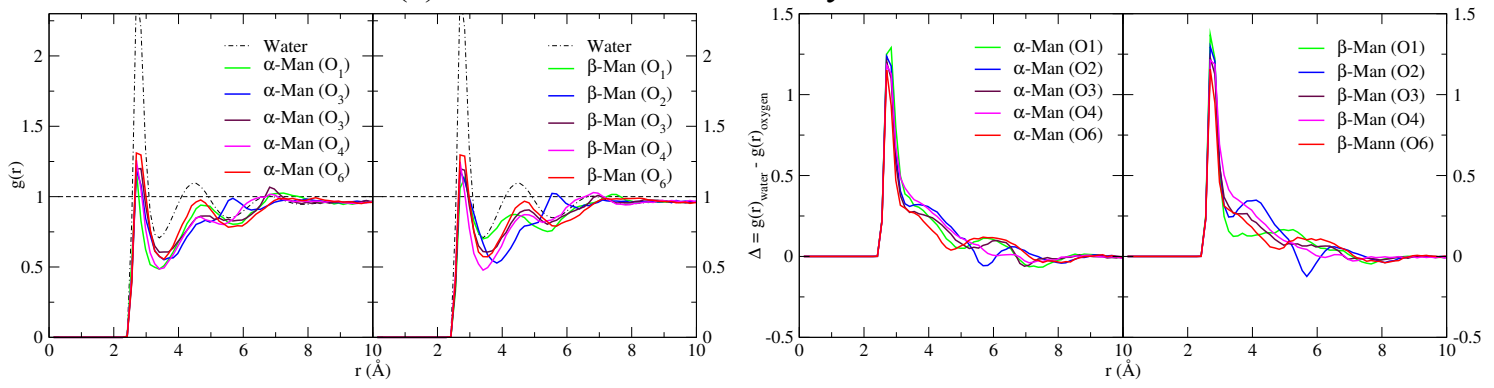

(d) Man - SWM4 drude system
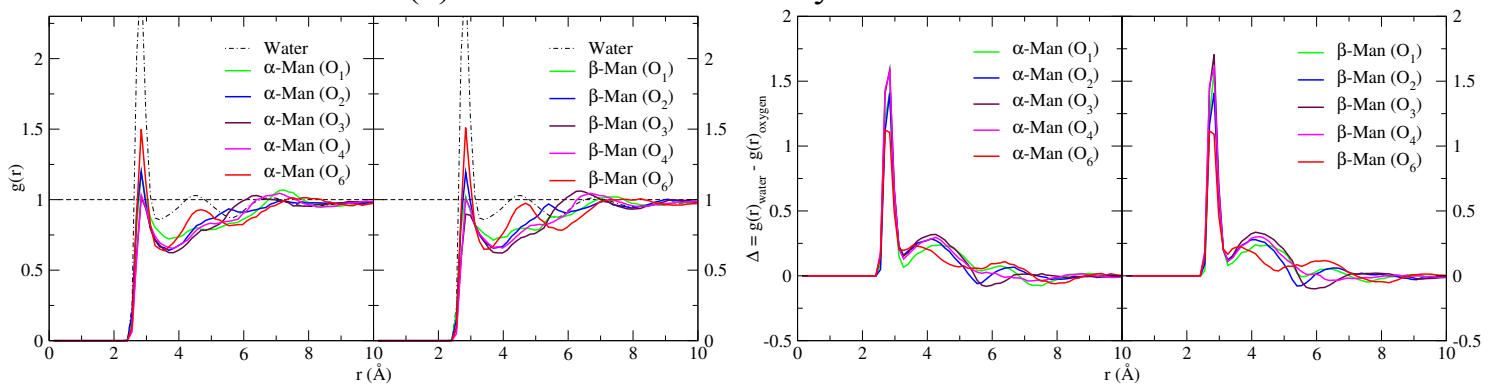

Figure S2: Left Panel: Selected oxygen (carbohydrate) - oxygen (water) radial distribution functions, $g(r)$, from $300 \mathrm{~K}$ simulations of carbohydrate (Man) in four water models (a) TIP3P, (b) TIP4P, (c) TIP5P and (d) SWM4. Right Panel: Differences $(\Delta)$ between the radial distribution functions. $\Delta$ is calculated as the differences between $g(r)$ of pure water and $g(r)$ of selected oxygen carbohydrate (Man) - oxygen (water) radial distribution from $300 \mathrm{~K}$ simulations. 


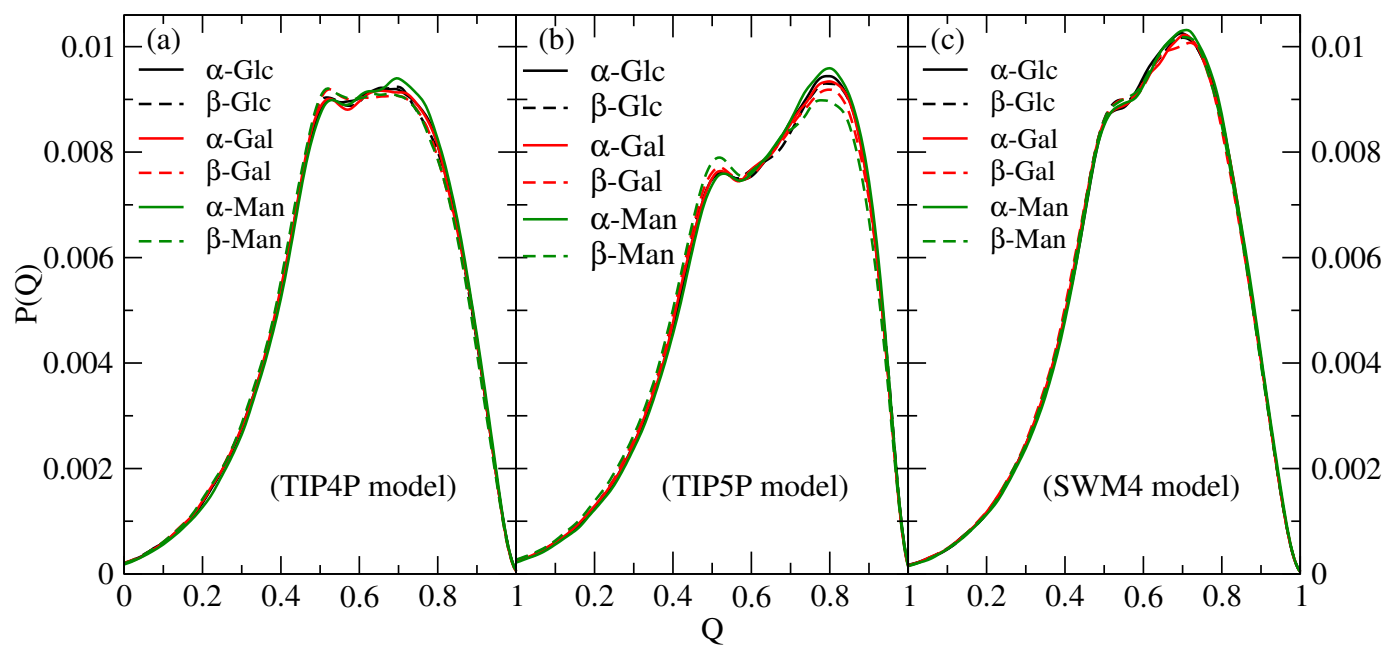

Figure S3: The $P(Q)$ distribution of water molecules in the first solvation shell (less than $3.5 \AA$ ) around carbohydrates from the respective water model simulations (a) TIP4P, (b) TIP5P and (c) SWM4 simulations at $300 \mathrm{~K}$. 

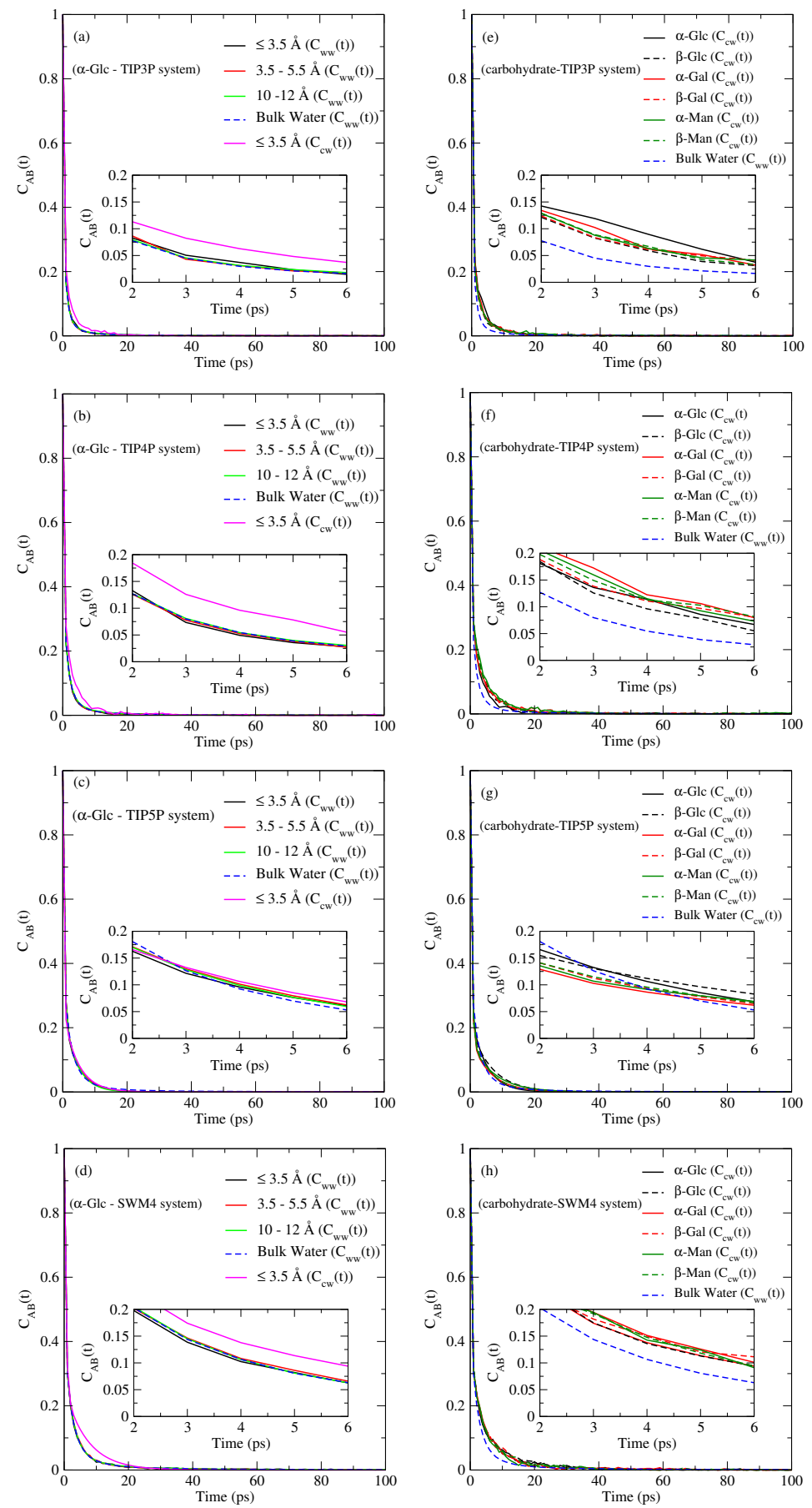

Figure S4: Left Panel: Comparison of intermittent H-bond autocorrelation function $\mathrm{C}_{\mathrm{ww}}$ in the three hydration shells from (a) $\alpha-$ Glc - TIP3P, (b) $\alpha-$ Glc - TIP4P, (c) $\alpha-$ Glc - TIP5P, (d) $\alpha-$ Glc - SWM4 simulations at $300 \mathrm{~K}$. The water-water $\left(C_{w w}\right)$ autocorrelation function from bulk water simulations and the carbohydrate water intermittent H-bond autocorrelation function $C_{c w}$ for the first hydration shell are also presented for comparison. Right Panel: Carbohydrate water intermittent H-bond autocorrelation function $\mathrm{C}_{\mathrm{cw}}$ for the first hydration shell of the $\alpha$ and $\beta$ anomers of Glc, Gal and Man from the (e) TIP3P, (f) TIP4P, (g) TIP5P and (h) SWM4 simulations. $C_{w w}$ from bulk water simulations is also presented for comparison. 

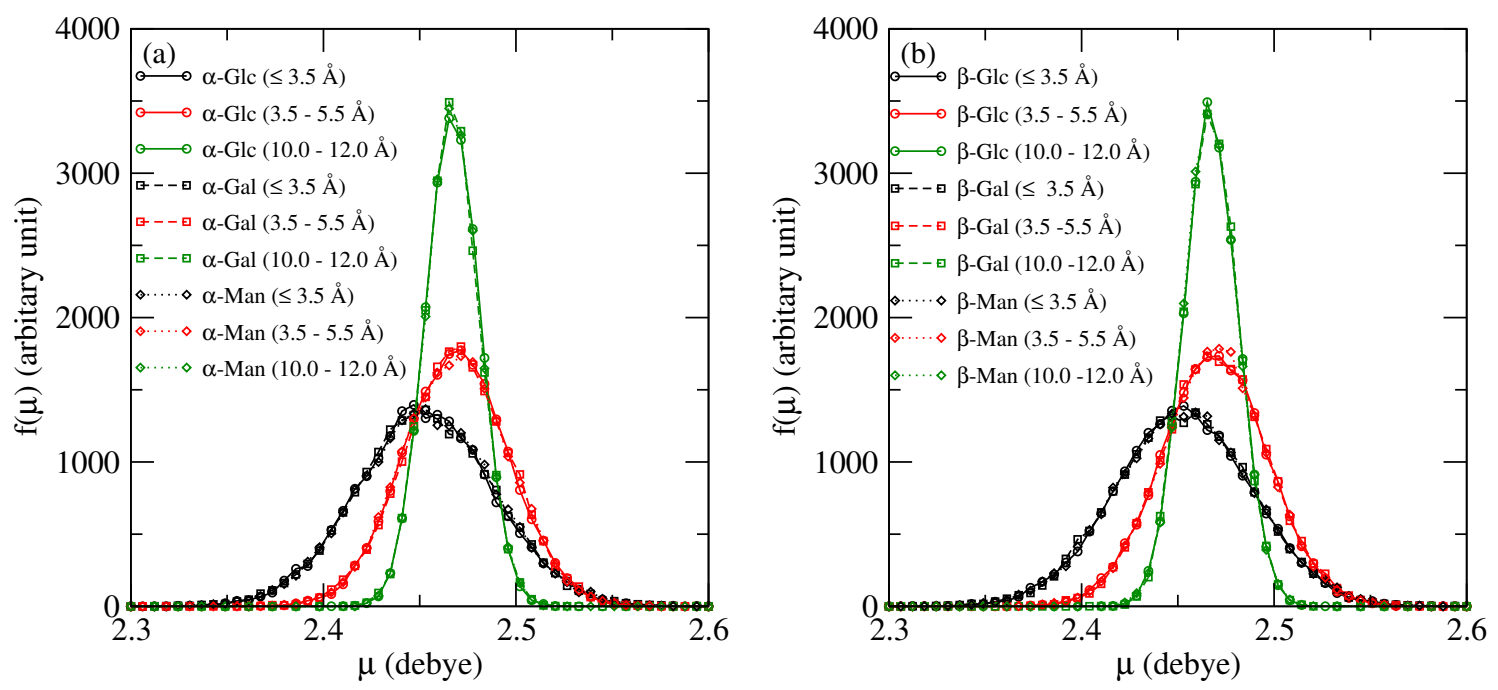

Figure S5: Dipole moment distribution of water molecules around the (a) $\alpha$ and (b) $\beta$ anomers of carbohydrates from the carbohydrate water SWM4 simulations at $300 \mathrm{~K}$. 

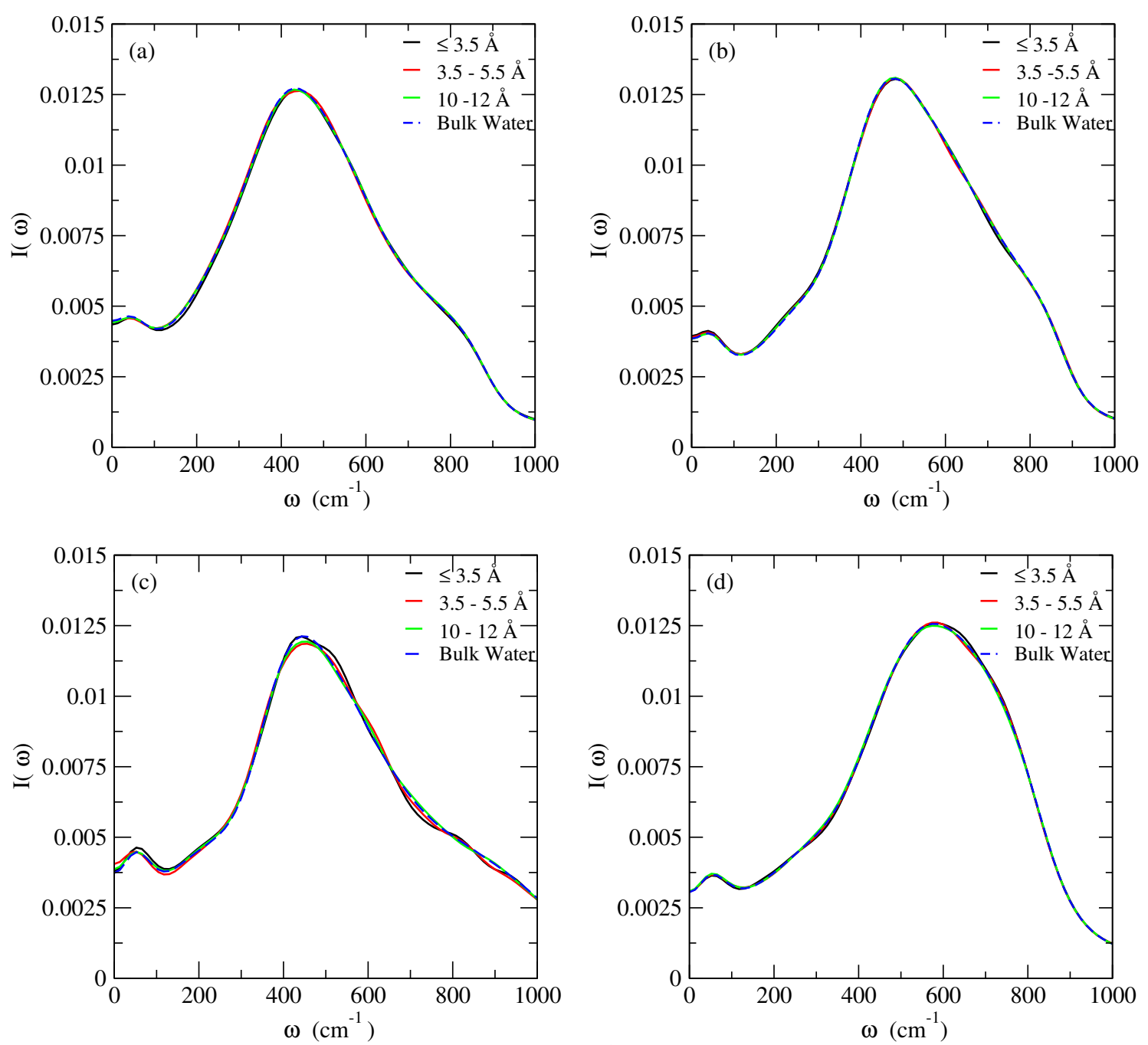

Figure S6: Vibrational spectra in the $0-1000 \mathrm{~cm}^{-1}$ range for hydrogen atoms of water molecules from the (a) $\alpha$-Glc - TIP3P, (b) $\alpha$-Glc - TIP4P, (c) $\alpha$-Glc - TIP5P, (d) $\alpha$ Glc - SWM4 simulations at 300K. 

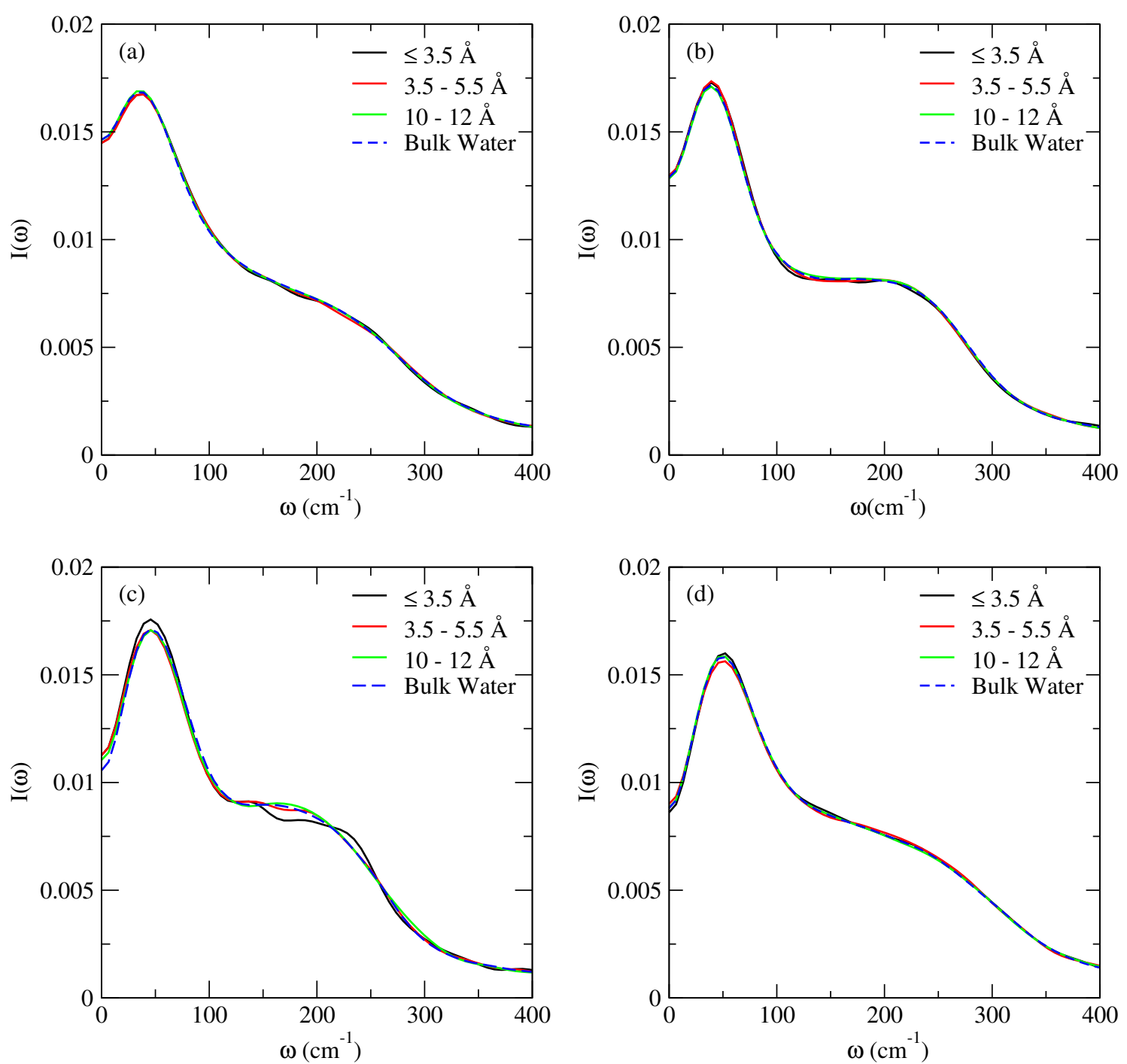

Figure S7: Vibrational spectra in the $0-400 \mathrm{~cm}^{-1}$ range for oxygen atoms of water molecules from the (a) $\alpha$-Glc - TIP3P, (b) $\alpha$-Glc - TIP4P, (c) $\alpha$-Glc - TIP5P, (d) $\alpha$ Glc - SWM4 simulations at 300K. 
Table S1: Fractional Populations of the Conformers of the Exocyclic Torsion $\left(\mathrm{O}_{5}-\mathrm{C}_{5^{-}}\right.$ $\left.\mathrm{C}_{6}-\mathrm{O}_{6}=\omega\right)$.

\begin{tabular}{|c|c|c|c|c|}
\hline \multicolumn{5}{|c|}{ Carbohydrate-TIP3P system } \\
\hline \multirow{2}{*}{ Conformers } & \multicolumn{2}{|r|}{ Glucose } & \multicolumn{2}{|c|}{ Galactose } \\
\hline & MD & Experiments $^{\mathrm{a}}$ & MD & Experiments $^{\mathrm{a}}$ \\
\hline \multirow[t]{2}{*}{$\mathrm{GG}\left(\omega=-60^{\circ}\right)$} & 0.30 & 0.40 & 0.01 & 0.03 \\
\hline & 0.05 & 0.31 & 0.01 & 0.03 \\
\hline \multirow{2}{*}{ GT $\left(\omega=60^{\circ}\right)$} & 0.65 & 0.53 & 0.57 & 0.74 \\
\hline & 0.87 & 0.61 & 0.53 & 0.72 \\
\hline \multirow[t]{2}{*}{$\mathrm{TG}\left(\omega=180^{\circ}\right)$} & 0.05 & 0.07 & 0.42 & 0.23 \\
\hline & 0.08 & 0.08 & 0.46 & 0.25 \\
\hline \multicolumn{5}{|c|}{ Carbohydrate-TIP4P system } \\
\hline \multirow[t]{2}{*}{ Conformers } & \multicolumn{2}{|r|}{ Glucose } & \multicolumn{2}{|c|}{ Galactose } \\
\hline & MD & Experiments $^{\mathrm{a}}$ & MD & Experiments $^{\mathrm{a}}$ \\
\hline \multirow[t]{2}{*}{$\mathrm{GG}\left(\omega=-60^{\circ}\right)$} & 0.48 & 0.40 & 0.07 & 0.03 \\
\hline & 0.22 & 0.31 & 0.05 & 0.03 \\
\hline \multirow{2}{*}{$\operatorname{GT}\left(\omega=60^{\circ}\right)$} & 0.45 & 0.53 & 0.49 & 0.74 \\
\hline & 0.70 & 0.61 & 0.49 & 0.72 \\
\hline \multirow[t]{2}{*}{$\mathrm{TG}\left(\omega=180^{\circ}\right)$} & 0.10 & 0.07 & 0.44 & 0.23 \\
\hline & 0.08 & 0.08 & 0.46 & 0.25 \\
\hline \multicolumn{5}{|c|}{ Carbohydrate-TIP5P system } \\
\hline \multirow[t]{2}{*}{ Conformers } & \multicolumn{2}{|r|}{ Glucose } & \multicolumn{2}{|c|}{ Galactose } \\
\hline & MD & Experiments $^{\mathrm{a}}$ & MD & Experiments $^{\mathrm{a}}$ \\
\hline \multirow[t]{2}{*}{$\mathrm{GG}\left(\omega=-60^{\circ}\right)$} & 0.70 & 0.40 & 0.01 & 0.03 \\
\hline & 0.53 & 0.31 & 0.02 & 0.03 \\
\hline \multirow[t]{2}{*}{$\operatorname{GT}\left(\omega=60^{\circ}\right)$} & 0.28 & 0.53 & 0.50 & 0.74 \\
\hline & 0.45 & 0.61 & 0.38 & 0.72 \\
\hline \multirow{2}{*}{$\mathrm{TG}\left(\omega=180^{\circ}\right)$} & 0.02 & 0.07 & 0.49 & 0.23 \\
\hline & 0.14 & 0.08 & 0.60 & 0.25 \\
\hline
\end{tabular}

\begin{tabular}{cclll}
\hline & \multicolumn{3}{c}{ Carbohydrate-SWM4 system } \\
\hline \multirow{2}{*}{ Conformers } & \multicolumn{3}{c}{ Glucose } & \multicolumn{3}{c}{ Galactose } \\
\cline { 2 - 5 } & MD & Experiments $^{\mathrm{a}}$ & MD & Experiments $^{\mathrm{a}}$ \\
\hline GG $\left(\omega=-60^{\circ}\right)$ & 0.18 & 0.40 & 0.16 & 0.03 \\
& 0.18 & 0.31 & 0.17 & 0.03 \\
GT $\left(\omega=60^{\circ}\right)$ & 0.71 & 0.53 & 0.68 & 0.74 \\
& 0.68 & 0.61 & 0.60 & 0.72 \\
TG $\left(\omega=180^{\circ}\right)$ & 0.11 & 0.07 & 0.16 & 0.23 \\
& 0.14 & 0.08 & 0.23 & 0.25 \\
\hline
\end{tabular}

${ }^{a}$ Thibaudeau, C.; Stenutz, R.; Hertz, B.; Klepach, T.; Zhao, S.; Wu, Q. Q.; Carmichael, I.; Serianni, A. S. J Am Chem Soc 2004, 126, 15668. 
Table S2: Coordination numbers of water oxygen $\left(\mathrm{O}_{\mathrm{w}}\right)$ around the carbohydrate oxygen $\left(\mathrm{O}_{\mathrm{n}}\right)$ by integrating the $\mathrm{O}_{\mathrm{n}}-\mathrm{O}_{\mathrm{w}}$ radial distribution function to the first minima of the $\mathrm{g}(\mathrm{r})$ distribution.

\begin{tabular}{lllllllll}
\hline \multirow{2}{*}{ Oxygen } & \multicolumn{7}{c}{ Glucose } \\
\cline { 2 - 9 } & TIP3P & TIP4P & TIP5P & SWM4 & TIP3P & TIP4P & TIP5P & SWM4 \\
\hline $\mathrm{N}_{1}$ & 2.65 & 2.28 & 2.15 & 3.12 & 3.17 & 3.12 & 2.59 & 3.09 \\
$\mathrm{O}_{2}$ & 3.35 & 2.50 & 2.82 & 2.87 & 2.90 & 2.86 & 2.41 & 2.42 \\
$\mathrm{O}_{3}$ & 2.90 & 2.11 & 2.12 & 3.33 & 2.50 & 2.43 & 2.41 & 3.34 \\
$\mathrm{O}_{4}$ & 2.25 & 1.93 & 1.89 & 2.67 & 2.23 & 1.90 & 1.87 & 3.50 \\
$\mathrm{O}_{6}$ & 2.68 & 2.23 & 2.11 & 2.24 & 2.68 & 2.25 & 2.14 & 2.54 \\
\hline
\end{tabular}

\begin{tabular}{|c|c|c|c|c|c|c|c|c|}
\hline \multirow{3}{*}{ Oxygen } & \multicolumn{8}{|c|}{ Galactose } \\
\hline & \multicolumn{4}{|c|}{$\alpha$} & \multicolumn{4}{|c|}{$\beta$} \\
\hline & TIP3P & TIP4P & TIP5P & SWM4 & TIP3P & TIP4P & TIP5P & SWM4 \\
\hline $\mathrm{O}_{1}$ & 2.67 & 2.28 & 2.19 & 2.71 & 3.20 & 2.74 & 2.61 & 3.58 \\
\hline $\mathrm{O}_{2}$ & 2.94 & 2.50 & 2.88 & 2.82 & 2.53 & 2.48 & 2.10 & 2.43 \\
\hline $\mathrm{O}_{3}$ & 2.93 & 2.46 & 2.15 & 3.78 & 2.52 & 2.45 & 2.80 & 3.34 \\
\hline $\mathrm{O}_{4}$ & 2.88 & 2.20 & 2.79 & 2.82 & 2.56 & 2.52 & 2.51 & 2.83 \\
\hline $\mathrm{O}_{6}$ & 2.29 & 2.57 & 2.11 & 2.18 & 2.63 & 2.20 & 2.08 & 2.14 \\
\hline \multirow{3}{*}{ Oxygen } & \multicolumn{8}{|c|}{ Mannose } \\
\hline & \multicolumn{4}{|c|}{$\alpha$} & \multicolumn{4}{|c|}{$\beta$} \\
\hline & TIP3P & TIP4P & TIP5P & SWM4 & TIP3P & TIP4P & TIP5P & SWM4 \\
\hline $\mathrm{O}_{1}$ & 1.89 & 2.18 & 1.71 & 2.98 & 3.17 & 3.13 & 2.19 & 2.87 \\
\hline $\mathrm{O}_{2}$ & 2.75 & 2.71 & 2.33 & 2.80 & 3.22 & 3.16 & 3.22 & 2.85 \\
\hline $\mathrm{O}_{3}$ & 2.53 & 2.44 & 2.14 & 2.99 & 3.30 & 2.45 & 2.45 & 3.38 \\
\hline $\mathrm{O}_{4}$ & 1.99 & 1.91 & 1.88 & 3.06 & 2.26 & 1.92 & 1.87 & 3.51 \\
\hline $\mathrm{O}_{6}$ & 2.67 & 2.63 & 2.51 & 2.53 & 2.68 & 2.62 & 2.49 & 2.22 \\
\hline
\end{tabular}


Table S3: Variation of dipole moment of water molecules in the hydration shell of six hexopyranose-water system.

\begin{tabular}{|c|c|c|c|}
\hline Molecule & Hydration shell & Mean dipole value & Standard daviation \\
\hline \multirow{4}{*}{$\alpha-G l c$} & $\leq 3.5 \AA$ & 2.45 & 0.04 \\
\hline & $3.5-5.5 \AA$ & 2.47 & 0.03 \\
\hline & $10.0-12.0 \AA$ & 2.46 & 0.01 \\
\hline & $\leq 3.5 \AA$ & 2.45 & 0.04 \\
\hline \multirow{3}{*}{$\beta$-Glc } & $3.5-5.5 \AA$ & 2.47 & 0.03 \\
\hline & $10.0-12.0 \AA$ & 2.46 & 0.01 \\
\hline & $\leq 3.5 \AA$ & 2.45 & 0.04 \\
\hline \multirow[t]{3}{*}{$\alpha-\mathrm{Gal}$} & $3.5-5.5 \AA$ & 2.47 & 0.03 \\
\hline & $10.0-12.0 \AA$ & 2.46 & 0.01 \\
\hline & $\leq 3.5 \AA$ & 2.45 & 0.04 \\
\hline \multirow[t]{3}{*}{$\beta-\mathrm{Gal}$} & $3.5-5.5 \AA$ & 2.47 & 0.03 \\
\hline & $10.0-12.0 \AA$ & 2.46 & 0.01 \\
\hline & $\leq 3.5 \AA$ & 2.45 & 0.04 \\
\hline \multirow[t]{3}{*}{$\alpha$-Man } & $3.5-5.5 \AA$ & 2.47 & 0.03 \\
\hline & $10.0-12.0 \AA$ & 2.46 & 0.01 \\
\hline & $\leq 3.5 \AA$ & 2.45 & 0.04 \\
\hline \multirow[t]{2}{*}{$\beta$-Man } & $3.5-5.5 \AA$ & 2.47 & 0.03 \\
\hline & $10.0-12.0 \AA$ & 2.46 & 0.01 \\
\hline
\end{tabular}

